\title{
Models for Pop I stars: implications for age determinations
}

\author{
Georges Meynet ${ }^{1}$, Patrick Eggenberger ${ }^{2}$, Nami Mowlavi $^{1}$ and \\ André Maeder ${ }^{1}$ \\ ${ }^{1}$ Geneva University, Geneva Observatory \\ CH-1290 Versoix, Switzerland \\ email: georges.meynet@unige.ch \\ ${ }^{2}$ Institut d'Astrophysique et de Géophysique de l'Université de Liège, Allée du 6 Août, 17 \\ B-4000 Liège, Belgium eggenberger@astro.ulg.ac.be
}

\begin{abstract}
Starting from a few topical astrophysical questions which require the knowledge of the age of Pop I stars, we discuss the needed precision on the age in order to make progresses in these areas of research. Then we review the effects of various inputs of the stellar models on the age determination and try to identify those affecting the most the lifetimes of stars.
\end{abstract}

Keywords. convection, diffusion; Stars: rotation, mass loss, magnetic fields

\section{Importance of reliable age-calibrations for Pop I stars}

Pop I stars cover a wide range of ages, from millions to billions of years, and their age determination is useful for studying the evolution of processes with very different timescales, going from the evolution of planetary systems to the analysis of powerful starbursts in remote galaxies. A few examples are given below:

- How does star formation propagate around young star-forming regions? What are the timescales for star formation to trigger star formation (see for instance the very young associations observed in the vicinity of $\eta$ Car, discussed in the review by Smith \& Brooks 2008)? In order to study the time sequence between different associations, relative ages with an accuracy better than about $10-20 \%$ would be quite useful, and that means an absolute accuracy of a few 100,000 years on ages of a few Myr.

- What are the ages of young, powerful starbursts in remote galaxies? In distant galaxies, where individual stars cannot be resolved, emission-line ratios in the spectrum of the integrated light can be used to determine the age of starbursts (see the review by Leitherer 2005). The typical ages are of a few Myr and the needed precision is of the same order as the one indicated above for the age determination of resolved associations. Let us however stress that here, in addition to the uncertainties pertaining the stellar models, those due to the duration of the burst of star formation, to the stellar Initial Mass Function and to the possibility of superpositions of many starbursts makes the exercise still more difficult.

- What is the upper mass limit of the progenitors of White Dwarfs (WD)? To answer this question one needs to establish the initial-final mass relationship of WD. This can be done by determining the age of the open clusters where WD are observed. This age is then used, together with the cooling age of the WD, to estimate the mass of the WD progenitor using stellar models. In this process age determination enters in three ways: first through the isochrone fitting of the cluster, second through the cooling age of the WD and finally through an age-mass relation (Weidemann 2000; see also the talks by Kalirai and Richer in this volume). To determine the upper mass limit for the progenitor 
of WD, accurate determination of the age of clusters with a mass at the turn off around $8 \mathrm{M}_{\odot}$ are needed, which means ages of the order of a few tens of Myr. In this age range, an uncertainty of $20 \%$ on the age translates into an uncertainty of about $1 \mathrm{M}_{\odot}$ on the mass (thus an uncertainty of $\sim 10 \%$ on the mass).

- What is the lifetime of a very-hot Jupiter? These giant planets orbit their host stars with orbital periods below 3 days. Given their proximity to their host stars, these planets should undergo some degree of evaporation due to the heating by stellar UV photons. Some models predict a catastrophic destiny for these planets, being completely evaporated in a relatively short timescale. If true, very-hot Jupiter could then only be observed around the youngest stars. Is this the case? Melo et al. (2006) find that none of the stars studied in their paper seem to be younger than 0.5 Gyr. Only lower limits for most of the ages of these stars are obtained. To make progresses in this area, restricted ranges of ages for the planet host stars should be obtained.

- What was the evolution of the chemical gradients in the Milky Way? Nowadays the Milky Way presents a gradient $\mathrm{d} \lg (\mathrm{O} / \mathrm{H}) / \mathrm{dR}$ (where $R$ is the Galactocentric radius) of between $-0.07 \mathrm{dex} / \mathrm{kpc}$ and -0.04 (see e.g. Daflon \& Cunha 2004). Was this gradient steeper or shallower in the past? From a theoretical point of view the answer is uncertain. In order to answer such a question, one needs objects for which the metallicity and the age can be measured. Open clusters and planetary nebulae are the objects that can be used as probes of the largest part of the history of the Galaxy (from a time when its age was about 6 Gyr until today), while simultaneously spanning a wide range of Galactocentric radii. In order to improve our present knowledge of the evolution of this gradient, a precision better than one half Gyr should be obtained on the ages (precision of about $10 \%$ needed).

- What is the form of the age-metallicity relation for the thin disc stars of the Galaxy? For instance Nordström et al. (2004) have found a small change of the mean metallicity of the thin disk since its formation and a very substantial scatter in metallicity at all ages. Again an accuracy better than about $10 \%$ on the age would allow us to sharpen this picture.

While age determinations may reach an internal precision sometimes better than $10 \%$, systematic effects may prevent a similar level of accuracy being reached in absolute ages. In the following, we shall focus on the systematic effects on age estimates due to uncertainties pertaining to various physical effects accounted for in stellar models.

\section{Comparison of different models for near-solar metallicity}

In table 1 below, we list some grids of non-rotating stellar models, covering the case of Pop I stars (the list is not exhaustive and refers to only one paper per group!). To make a first comparison, we can plot, for different models, the relation between the bolometric magnitude at the end of the Main-Sequence phase and the corresponding age. This is done in the left panel of Fig. 1. Overall there is good agreement between the plotted models. The slope of the relation $\left[\mathrm{M}_{\mathrm{bol}} \propto \sim 15 / 4 \lg (\right.$ age $\left.)\right]$ agrees well with analytical estimates based on the mass-luminosity relation $\left(L \propto\left(\mu^{4} M^{3}\right) / \kappa\right.$, where $\mu$ is the mean molecular weight, $M$ the total mass and $\kappa$, the mean opacity inside the star) and the mass-age relation $\left(\tau_{\mathrm{MS}} \propto\left(q X M f c^{2}\right) /\left(\mu^{4} M^{3} / \kappa\right)\right.$, where $q$ is the mass fraction of the total mass of the star where nuclear reactions occur, $X$ the mass fraction of hydrogen, $c$ the velocity of light, $f$ the fraction of the initial mass of hydrogen transformed into energy when H-burning occurs, $f$ is equal to 0.007 ). Thus an error of 0.1 magnitude implies an error on the age of less than $3 \%$. 
Table 1. Some grids of non-rotating stellar models for near solar metallicity.

\begin{tabular}{lcccc}
\hline Reference & Masses & $Y$ & $Z$ & $\alpha_{\text {ov }}$ \\
\hline & & & & \\
Bono et al. $2000^{(1)}$ & $3-15$ & 0.27 & 0.02 & 0.0 \\
Claret 2004 & $0.8-125$ & 0.28 & 0.02 & 0.2 \\
Demarque et al. $2004^{(1)}$ & $4-52$ & 0.279 & 0.02 & $(6)$ \\
Dominguez et al. 1999 & $1.2-9$ & 0.28 & 0.02 & 0.0 \\
Dotter et al. $2007^{(1)}$ & $0.1-1.8$ & 0.274 & 0.0189 & 0.2 see $(3)$ \\
Girardi et al. $2000^{(1)}$ & $0.15-7$ & 0.273 & 0.019 & $(5)$ \\
Pietrinferni et al. $2004^{(1)}$ & $0.5-10$ & 0.273 & 0.0198 & 0.2 see $(3)$ \& (4) \\
Schaller et al. $1992^{(1)}$ & $0.8-120$ & 0.30 & 0.02 & 0.2 \\
VandenBerg et al. $2006^{(1)}$ & $0.5-2.4$ & 0.2715 & 0.0188 & see $(2)$ \\
Ventura et al. 1998 & $0.6-15$ & 0.274 & 0.017 & FST $(7)$ \\
\hline
\end{tabular}

1 Models for other compositions are available in that paper.

2 Roxburgh criterion calibrated using binaries and clusters.

3 Value adopted in models with well developed convective cores.

Lower values are used for small convective cores see Dotter et al. (2007)

4 Models without overshooting with the same initial composition are also available.

5 The overshooting is accounted for using the formalism of Bressan et al. (1981).

6 The overshooting is accounted for using the formalism of Demarque et al. (2004).

7 Convection is treated according to the Full Spectrum Turbulence model.
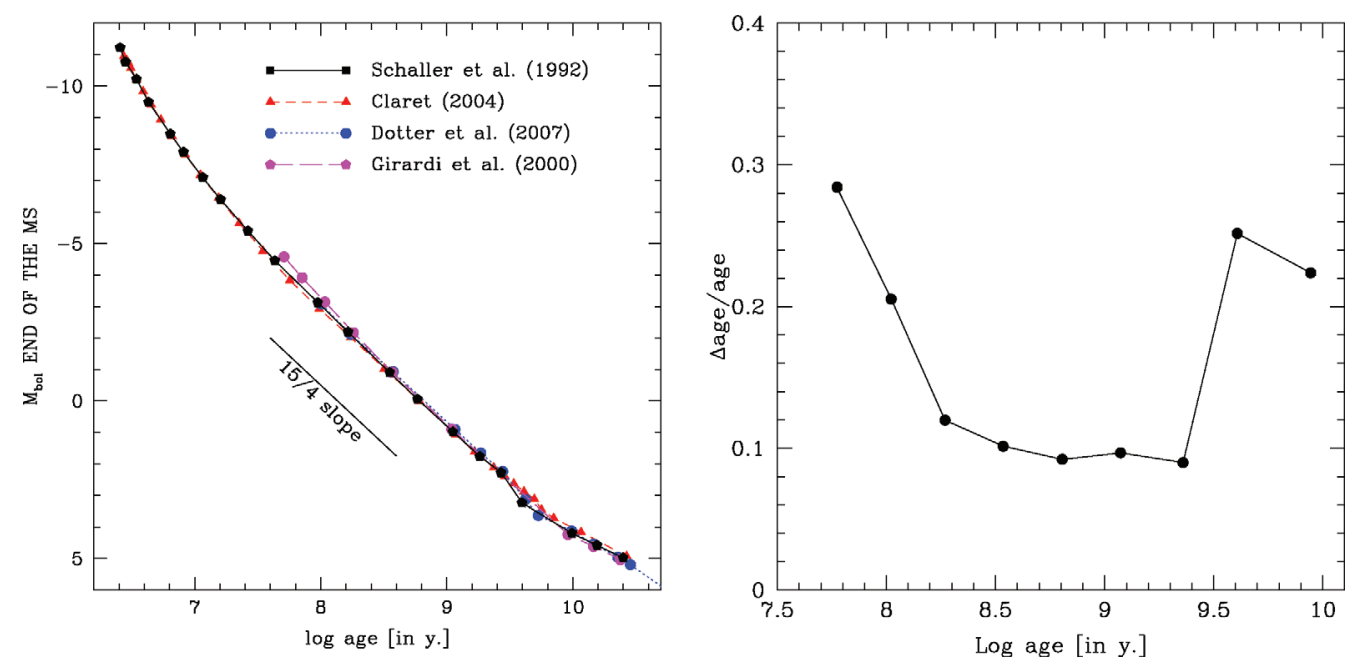

Figure 1. Left panels: bolometric magnitude at the turn-off versus the age, for different non-rotating stellar models with similar physical ingredients. Right panel: dispersion of the ages due to the use of the different stellar models listed on the left panel.

In the right panel of Fig. 1, we indicate the difference between the minimum age and the maximum age given by these models for a given magnitude, normalised to the mean age, the mean age being (maximum - minimum age) $/ 2$. We see that the age dispersion is of the order of $10 \%$ for ages between $200 \mathrm{Myr}$ and 2 Gyr. The dispersion increases up to values between $25 \%$ and $30 \%$ for greater and smaller ages. Most of the differences come from different ways of treating the way the overshooting parameter evolves as a function of the initial mass (see the respective references describing the models).

\section{Effects of a change in the initial abundances}

In non-rotating models, the metallicity affects the evolution of stars mainly through its impact on the radiative opacities, the equation of state and the nuclear reaction rates. 

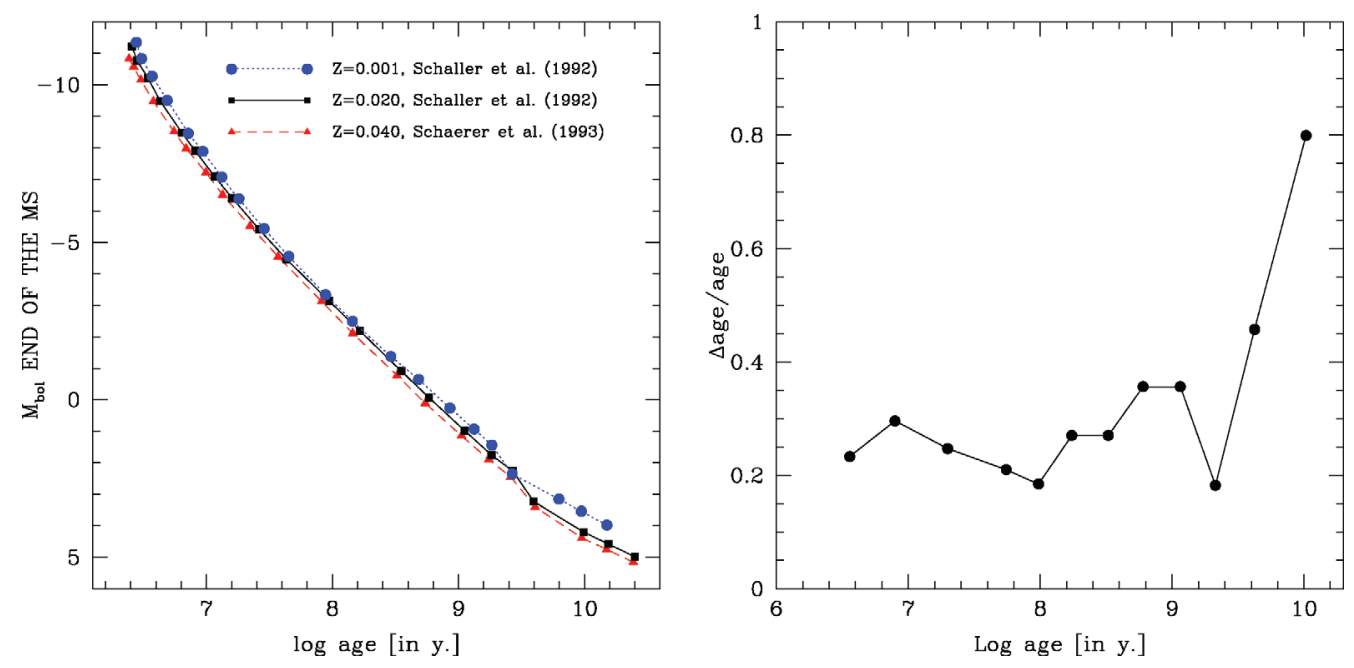

Figure 2. Left panels: bolometric magnitude at the turn off versus the age, for non-rotating stellar models at different metallicities. Right panel: dispersion of the ages due to a change of metallicity (see left panel for the list of models used).

For stars with initial mass greater than about $30 \mathrm{M}_{\odot}$, mass loss becomes an important ingredient already during the MS phase and the effects of metallicity on the mass-loss rates have to be taken into account (see the effect on age determination below). These effects of metallicity on stellar models are discussed in detail in Mowlavi et al. (1998). Metallicity also affects the transport mechanisms induced by rotation (Maeder \& Meynet 2001). Typically a star of given initial mass, starting its evolution with a given initial rotation velocity, will be more efficiently mixed by rotation at low metallicity than at high metallicity.

The most important effect of a change of metallicity occurs through the effect on the opacity (at least for a large range of metallicities and ages). In general, the opacities increase with increasing metallicity. This is the case for opacity due to bound-free and free-free transitions. Using the mass-luminosity relation seen above, one immediately deduces that the increase of the opacity produces a decrease of the luminosity of a given initial mass model. In contrast, for massive stars free-electron scattering is the main opacity source. This opacity depends only on $X\left[\kappa_{e} \simeq 0.20(1+X)\right]$, which is approximately constant for $Z \leqslant 0.01$ and decreases with increasing $Z$ at $Z \geqslant 0.01$. Thus at high $Z$ the luminosity of a massive star increases with metallicity.

In Fig. 2 (left panel) the relation between the bolometric magnitude at the turn off and age are shown, for various metallicities (see references on the figure). When the metallicity increases, a given magnitude is achieved by a higher initial stellar mass, since, for the $\mathrm{Z}$ range considered here, the luminosity of the tracks for a given initial mass are decreased. Thus smaller ages are obtained at higher $\mathrm{Z}$ for a given value of the turn-off magnitude. In Fig. 2 (right panel) we have plotted the age dispersion which would result from using tracks corresponding to different initial metallicities in the range between $\mathrm{Z}=0.001$ and $\mathrm{Z}=0.040$ (i.e. for $[\mathrm{Fe} / \mathrm{H}]$ between about -1.3 and +0.3 ). We see that the dispersion is higher than that resulting from different stellar grids with similar metallicities. It amounts to about $30 \%$ for ages below about 3 Gyr. It increases a lot for greater values of the ages, reflecting the increased sensitivity of the luminosity on $\mathrm{Z}$ in the low-mass range. This demonstrates that precise determination of the age needs relatively precise determination of the metallicity. 
Dotter et al. (2007) have recently studied the impact of individual changes in the abundances of some elements. They computed models for stars with masses between $0.5 \mathrm{M}_{\odot}$ and $3 \mathrm{M}_{\odot}$ enhancing the abundance of one element, keeping $\mathrm{X}, \mathrm{Y}$ and $\mathrm{Z}$ constant. Of course enhancing one element, at constant Z, must be done at the expense of all other elements. This work shows that the elements which have the most important effects are oxygen and iron. Varying their abundances by a factor of two produces changes in the MS lifetime, for a star of given initial mass, of $15 \%$ (decrease for $\mathrm{O}$ and increase for $\mathrm{Fe}$, see their Fig. 13). Let us note that the mass fraction of helium also has a big impact on the structure and the evolution of stars. For instance, models by Claret (1997) or Bono et al. (2000) have been computed with different He mass fractions. We refer the reader to these works for more details on that question (see also the paper by Decressin et al. in the present volume).

\section{Treatment of convection}

The treatment of convection, and more generally of all turbulent processes, remains one of the biggest difficulties in stellar modeling. Depending on the criterion chosen for the set up of the convective instabilities (Schwarzschild or Ledoux criterion), on the efficiency of semiconvection, and on the amplitude of the overshooting effects, the quantity of fuel, the luminosity, and therefore the MS lifetime, can vary a lot. For instance, a moderate overshoot $\left(0.2 \mathrm{H}_{P}\right)$ at the border of the Schwarzschild convective core associates an age at turn off luminosities equal to 2 [ $\left.\log \mathrm{L} / \mathrm{L}_{\odot}\right]$ (turn off mass around $\left.3 \mathrm{M}_{\odot}\right), 1\left(1.6 \mathrm{M}_{\odot}\right.$ ) and $0.5\left(1.3 \mathrm{M}_{\odot}\right)$ which are respectively $40 \%$, a factor 2.5 and a factor 3.2 greater than the lifetimes obtained from models without overshoot. One sees that compared to the age dispersion arising from different grids of models (but with similar physical ingredients) or from grids at various metallicities, the age dispersion due to overshoot is much greater, especially in the low-mass range.

It has to be noted that the luminosity at the turn off is particularly sensitive to the amount of overshoot. This property leads many authors to use the width of the observed MS to calibrate the overshoot (see for instance Maeder \& Meynet 1989). Note that the temperature of the MS termination is much less sensitive to overshoot. This is due to the fact that overshooting increases the age but also pushes the MS termination to the red, in such a way that the $\log \mathrm{T}_{\text {eff }}$ at the MS termination versus age relation is nearly unchanged (see Figs 17 \& 18 in Maeder \& Meynet 1989). However, if the overshoot does not change the age assigned to a given turn-off effective temperature, it does affect the initial mass associated to it!

Asteroseismology will likely help to resolve the question of the size of the convective core in massive stars. Some first results are presented in Aerts (2008): in five B-type stars, the size of the convective core has been deduced from asteroseismology, and an overshoot parameter between 0.10 and $0.44 \mathrm{H}_{P}$ has been found.

In low-mass stars, non-adiabatic convection occurs in the outer layers. The extent of the convective zone is governed by the choice of the mixing length parameter $\alpha$, the value of it being fixed by calibrating solar models and/or from the position of the red giant branch in the HR diagram. Of course (as for the overshooting parameter) $\alpha$ is not a fundamental constant of nature and it may vary with mass, metallicity, and even the evolutionary phase. Thus it would be advantageous to have a means of constraining this quantity in other objects. Asteroseismology may be the tool to do that. Eggenberger et al. (2008) show that the observed parameters of 70 Oph A can be reproduced by two sets of very different models: one with an initial helium value of 0.266 , a mixing length parameter equal to 1.7998 (obtained from a solar model) and an age of 6.2 Gyr, another 
with an initial helium value of 0.240 , a mixing length parameter equal to 2.2497 (previous value multiplied by 1.25 ) and an age of 10.5 Gyr. Thus we see that the uncertainties on $\alpha$ may have a large impact on the age determination. While these two models show the same mean large separations, the mean small separation of the model with the higher initial helium abundance is significantly larger $(3 \mu \mathrm{Hz})$ than the one of the model with the lower initial helium abundance. Thus having a precise observed value of the mean small separation will allow us to obtain an independent determination of the age, of the mixing-length parameter and of the helium abundance.

\section{Rotation}

Rotation affects all the outputs of the stellar models and in particular changes the age derived for a star of given initial mass (Heger \& Langer 2000; Meynet \& Maeder 2000). By inducing internal mixing, rotation modifies both the total quantity of fuel available and the luminosity. The amplitude of the changes depends on the nature of the instabilities (induced by rotation) which are considered in the model. The two most important instabilities are shear instabilities and meridional currents. They both transport chemical species and angular momentum. They are much less efficient in transporting energy since their timescale is in general longer than the thermal diffusion timescale (see Zahn 1992).

Before discussing the implications of rotation on the age determinations, let us say a few words about the observations which can be used to constrain rotational mixing. A rotating star is predicted to show some nitrogen surface enrichment already during the main sequence. The amplitude of the nitrogen enrichment at the surface depends on the initial mass (increases with the mass), the age (increases with the age) and the initial rotational velocity. This is correct as long as we consider stars with a given initial composition (rotational mixing is more efficient at low Z) and whose evolution is not affected by a close binary companion. Thus we see that the nitrogen surface abundance is a function of at least three parameters: mass, age and velocity. To see a relation between $\mathrm{N}$-enrichment and velocity, it is necessary to use stars with different rotational velocities but having similar masses and ages.

When data samples limited in mass and ages are used, a very nice correlation is found between the surface N-enrichment and $v \sin i$ (see Figs. 3 and 4 in Maeder et al. 2008), supporting a N-enrichment dependence on rotational velocities. Stars beyond the end of the MS phase do not follow such a relation, since their velocities converge to low values (see Fig. 12 by Meynet \& Maeder 2000). A fraction, which we estimate to be $\sim 20 \%$ of the stars, may deviate from the relation as a result of binary evolution, either by tidal mixing or mass transfer.

Rotation modifies the size of the convective core (see Fig. 3 left panel). First the size of the convective core is decreased when rotation is accounted for. Indeed, due to the action of the centrifugal acceleration, the star behaves as one with a lower gravity, or as a star of lower initial mass. Then, when evolution proceeds, rotational diffusion will supply the core with hydrogen and the radiative envelope with helium. The result will be an increase in both the luminosity and the size of the convective core. The MS lifetimes are increased by rotation. During the MS phase, towards the turn-off, the tracks for a given mass in the HR diagram become more luminous and extend further to the cool part. In that respect, the effects of rotation are somewhat similar to that of overshooting (but of course the effects of rotation cannot be modeled by adding overshooting; for instance, as indicated above, rotation modifies the surface abundances, while overshooting does not). A given width of the MS can be obtained by a combination of rotation velocity and overshooting parameter (see Talon et al. 1997). For example, in the temperature range 

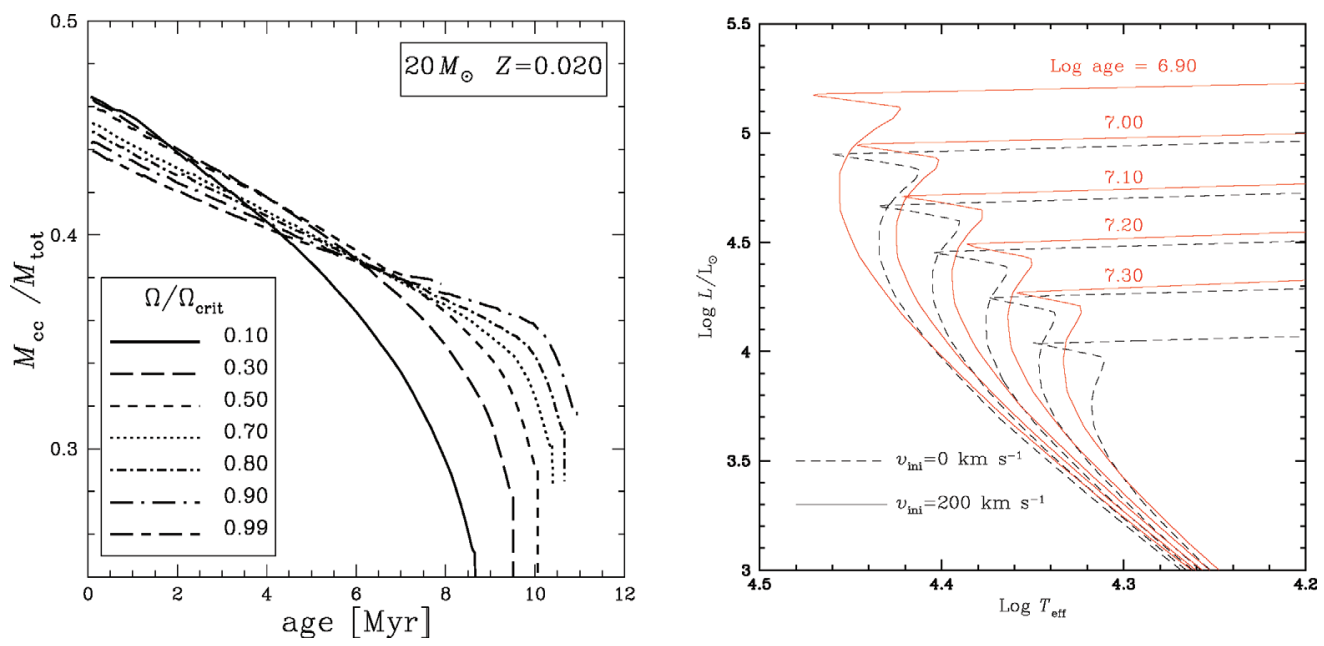

Figure 3. Left panels: Evolution of the size of the convective core during the MS, for the various values of rotational rate, in a $20 \mathrm{M}_{\odot}$ star, at standard metallicity. Figure taken from Ekström et al. (2008). Right panel: Isochrones computed from stellar evolutionary tracks for solar metallicity. The dashed and continuous lines correspond to the case of non-rotating and rotating stellar models respectively. In this last case, models have an initial velocity $v_{\text {ini }}$ of 200 $\mathrm{km} \mathrm{s}^{-1}$. The logarithms of the ages (in years) label the isochrones computed from the models with rotation. Figure taken from Meynet \& Maeder (2000)

between $\log T_{\text {eff }}=4.1$ and 4.3 (i.e. in the mass range between about 5 and $20 \mathrm{M}_{\odot}$ ), a similar MS width can be obtained either by non-rotating models with an overshooting parameter value of 0.2 , or by rotating models with an average rotational velocity on the MS around $100 \mathrm{~km} \mathrm{~s}^{-1}$ and an overshooting parameter value of 0.1 (estimates based on the models discussed in Ekström et al. 2008). Rotation and overshooting thus concur to make the MS band wider. Calibrating the overshooting parameter in non-rotating stellar models, by adjusting the MS band-width thus overestimates it. How can we disentangle the two processes? Comparisons of the core size, obtained by asteroseismology, in stars of similar mass, metallicity and age but having different rotational velocity will give precious constraints. Interestingly, the asteroseismic signature of overshooting, producing a sharp chemical gradient at the border of the convective core, is different from the signature of the smoother gradient produced by rotational mixing (see Montalban et al. 2008). Another approach would be to calibrate the overshooting parameter using stars massive enough to have a convective core, but with a velocity distribution biased toward slow rotators. Typically, early type F-stars would match these conditions (masses between 1.3 and $1.5 \mathrm{M}_{\odot}$ ), although recent rotational velocity determinations (see Royer et al. 2007) show that the mean velocity of these stars is not as low as previously thought: according to Royer et al. (2007), the mean velocity of these stars is in the range of $150 \mathrm{~km} / \mathrm{s}$, instead of $80-100 \mathrm{~km} / \mathrm{s}$. Thus the effects of rotation are probably not completely absent (although much less developed than in massive stars). Another difficulty with this mass range comes from the fact that probably the overshooting parameter varies with mass, as has been found by many authors using non-rotating models (see e.g. Girardi et al. 2000; Demarque et al. 2004). Thus the problem is not so easily resolved and its solution should probably await more stringent constraints coming from asteroseismology (see the talk by Vauclair in the present volume).

To give an idea of the effects of rotation on the age determination of young clusters, Fig. 3, right panel, compares isochrones obtained from non-rotating and rotating models. 
The rotating models have a rotational velocity of $\sim 150 \mathrm{~km} / \mathrm{s}$, on average, during the MS phase. We see that the age determined from rotating models are $\sim 25 \%$ higher than those obtained from non-rotating ones (Meynet \& Maeder 2000).

When the rotation is high enough, significant changes in the shape of the star are expected. Typically, for a $20 \mathrm{M}_{\odot}$ star, when $\Omega / \Omega_{c}>0.7$ (average velocity on the MS superior to $280 \mathrm{~km} / \mathrm{s}$ ), the equatorial radius is more than $10 \%$ larger than the polar radius. The von Zeipel theorem (1924) then implies that the radiative flux in the polar region is higher than in the equatorial region. This can be translated into a change of the effective temperature with colatitude. A consequence is that fast rotators will be characterized by different values of the luminosity and of the effective temperature, depending on the viewing angle. For a fast rotator (initial velocities on the ZAMS such that $\Omega / \Omega_{\text {crit }} \sim 0.90$ ), the dispersion in age due to the dispersion in viewing angle can amount to about $40 \%$ (using the luminosity at the turn-off for determining the age, higher when the star is seen pole-on).

In a cluster there will be a distribution of rotational velocities and of the directions of the rotational axis. Thus we expect that the above effects will induce some dispersion in the position of the stars in the HR diagram around the turn off. Such effects should probably be accounted for when determining the age of clusters containing significant proportions of Be-stars (i.e. B-type stars with emission lines, where the emission is due to the presence of an expanding equatorial disk, whose origin is commonly attributed to the very fast rotation of the star). This is the case, for instance, in NGC 330, a cluster in the SMC (see e.g. Keller et al. 2000) in which $40 \%$ of the MS stars which are two magnitudes below the turn off are Be stars. Note that in the bin half-a-magnitude wide just below the turn-off, $80 \%$ of the stars are Be stars!

\section{Microscopic diffusion}

Microscopic diffusion appears as soon as a fluid is out of thermodynamic equilibrium. Any gradient of temperature, density, external forces like gravity or radiative forces, will induce a diffusion velocity. In most cases, the diffusion velocities are much weaker than other velocities in the medium, therefore microscopic diffusion can only have a significant effect in very stable media.

In non-rotating solar-mass models, microscopic diffusion tends to reduce the MS lifetime by about $10 \%$ (based on a model computed by P. Eggenberger for the present paper). The lifetime is reduced mainly because helium diffuses in the H-burning region, and hydrogen is pushed out from the core by mass conservation. Diffusion thus decreases the amount of available fuel for the star and thus its lifetime. When a small level of rotation is taken into account (initial velocity of about $50 \mathrm{~km} \mathrm{~s}^{-1}$ ), the lifetime increases, almost back to the same value as the model without diffusion and without rotation. This is due to the fact that the two effects nearly compensate, diffusion decreasing the quantity of fuel in the core and rotation increasing it. Eggenberger et al. (2008) find that accounting or not for the effect of diffusion changes the age estimate of the binary system $70 \mathrm{Oph} \mathrm{AB}$ (masses of about 0.9 and $0.7 \mathrm{M}_{\odot}$ ) by about $1 \mathrm{Gyr}$, a relative change of about $14-16 \%$.

\section{Mass loss}

The effects of mass loss during the MS phase begins to become significant for stars more massive than about $30 \mathrm{M}_{\odot}$, at solar metallicity (that means ages less than about $5 \mathrm{Myr}$ ). Mass loss makes a star of a given mass follow an evolution in the HR diagram similar to that of a star of a lower initial mass. This tends thus to reduce the luminosity and 
Table 2. Tentative estimates of the dispersion of ages obtained from models of different authors or computed with different physical ingredients.

\begin{tabular}{lccl}
\hline Cause & Range of ages & Relative error & Remarks \\
\hline & & & \\
Origin of stellar models & all & $10-30 \%$ & for similar input physics \\
metallicity & $6.5-9.5$ & $20-35 \%$ & for $-1.3<[\mathrm{Fe} / \mathrm{H}]<0.3$ \\
& $9.5-10.0$ & $35-80 \%$ & for $-1.3<[\mathrm{Fe} / \mathrm{H}]<0.3$ \\
changing $\mathrm{O}$ or Fe by a factor 2
\end{tabular}

to increase the MS lifetime. It may also make stars that are initially very massive enter into the WR phase early, even during the MS phase. The MS termination then occurs at younger ages and at lower luminosities than in the case without mass loss. Mass loss significantly affects the relationship between the magnitude at the main sequence turn-off and age, only for ages less than about 3.3 million years. High mass-loss rates reduces the age corresponding to a given value of the magnitude at the main sequence turn-off. For example, for $M_{b o l}$ at the turn-off equal to -10.8 , the difference between the ages derived using models with either normal or enhanced mass-loss rates amounts to $0.73 \mathrm{Myr}$, going from 3.55 Myr (normal mass-loss, Schaller et al. 1992) to 2.62 Myr (enhanced mass-loss, Meynet et al. 1994).

Depending on the intensity of mass loss, the evolution with time after a starburst episode of the fraction by number of WR to O-type stars varies. Thus an age determination based on the derivation of this fraction, estimated from emission-line ratios, is affected by uncertainties in the mass-loss rates. For instance, a young starburst showing a WR/O number ratio of 0.20 , at $\mathrm{Z}=0.02$, may have an age either of 4.25 or of $3.25 \mathrm{Myr}$, depending on whether standard or enhanced mass-loss rates are used, respectively (Meynet 1995). In the case of starbursts, other factors also have an impact on the age determination, such as the timescale for the star formation episode (in the example above, we supposed an instantaneous episode), or the slope of the IMF.

\section{Conclusions}

From Table 2 above we can estimate the accuracy which would be needed for various physical ingredients of the models, in order to reach, let's say, a $10 \%$ accuracy in the age. We see that a precision better than 0.12 dex in $[\mathrm{Fe} / \mathrm{H}]$ is needed to assure a $10 \%$ precision on the age, over the whole range of ages, and that individual abundances of oxygen and of iron should be known with an accuracy better than a factor of 2 . The size of the convective core should be known with a very high precision, especially in the lower mass range. As explained above, this question is intimately related to the effects of rotation. The value of $l / H_{P}$ should be known with an accuracy better that $5-10 \%$, and the mass-loss rate with a precision better than about $20 \%$.

As usual, improvements will be brought by progress both in observations and in our physical understanding of turbulence under stellar conditions. In the observational context, asteroseismology will play a key role. This technique allows us to probe the stellar interiors and provides, in addition to the classical constraints such as gravity and effective temperature, new observables, reducing significantly the possible range of values for 
the free parameters. From the point of view of theory, multi-dimensional computations of hydrodynamic processes like convection will provide more thoughtful recipes to take account of the effects of turbulence in stellar interiors.

The list of effects in Table 2 is not exhaustive: what are the consequences of magnetic fields, of internal gravity waves, of tidal mixing in close binaries, or of accretion during the pre-MS phase of massive stars? All these effects still need to be studied.

\section{References}

Aerts, C. 2008, in Massive Stars as Cosmic Engines, Proceedings of the International Astronomical Union, IAU Symposium, Volume 250, p. 237

Bono, G., Caputo, F., Cassisi, S., Marconi, M., Piersanti, L., \& Tornambe, A. 2000, ApJ, 543, 955

Claret, A. 1997, $A \& A S, 125,439$

Claret, A. 2004, $A \& A, 424,919$

Daflon, S. \& Cunha, K. 2004, ApJ, 617, 1115

Demarque, P., Woo, J.-H., Kim, Y.-C., \& Yi, S. K. 2004, ApJS, 155, 667

Dominguez, I., Chieffi, A., Limongi, M., \& Straniero, O. 1999, ApJ, 524, 226

Dotter, A., Chaboyer, B., Jevremovic', D., Baron, E., Ferguson, J. W., Sarajedini, A., \& Anderson, J. 2007a, AJ, 134, 376

Dotter, A., Chaboyer, B., Ferguson, J. W., Lee, H.-c., Worthey, G., Jevremovic', D., \& Baron, E. $2007 \mathrm{~b}, A p J, 666,403$

Eggenberger, P., Miglio, A., Carrier, F., Fernandes, J., \& Santos, N. C. 2008, A\&A, 482, 631

Ekström, S., Meynet, G., Maeder, A., \& Barblan, F. 2008a, A\&SA, 478, 467

Girardi, L., Bressan, A., Bertelli, G., \& Chiosi, C. 2000, A\&AS, 141, 371

Heger, A. \& Langer, N. 2000, ApJ, 544, 1016

Hunter, I., Brott, I., Lennon, D.J. et al. 2008, ApJ, 676, L29

Keller, S. C., Bessell, M. S., \& Da Costa, G. S. 2000, AJ, 119, 1748

Leitherer, C. 2005, in The Evolution of Starbursts: The 331st Wilhelm and Else Heraeus Seminar. AIP Conference Proceedings, Volume 783, p. 280

Maeder, A. \& Meynet, G. 1989, A\&BA, 210, 155

Maeder, A. \& Meynet, G. 2001, A\&BA, 373, 555

Maeder, A., Meynet, G., Ekstrom, S., \& Georgy, C. 2008, Comm. in Asteroseismology, Contribution to the Proceedings of the 38th LIAC, HELAS-ESTA, BAG, in press (arXiv:0810.0657)

Melo, C., Santos, N. C., Pont, F., Guillot, T., Israelian, G., Mayor, M., Queloz, D., \& Udry, S. 2006, A\&BA, 460, 251

Meynet, G. 1995, $A \mathscr{E} A, 298,767$

Meynet, G. \& Maeder, A. 2000, A\&BA, 361, 101

Meynet, G., Maeder, A., Schaller, G., Schaerer, D., \& Charbonnel, C. 1994, A\&AS, 103, 97

Montalban, J., Miglio, A., Eggenberger, P., \& Noels, A. 2008, Astronomische Nachrichten, 329, 535

Mowlavi, N., Meynet, G., Maeder, A., Schaerer, D., \& Charbonnel, C. 1998, A\& 4 A, 335, 573

Nordström, B., Mayor, M., Andersen, J., Holmberg, J., Pont, F., Jorgensen, B. R., Olsen, E. H., Udry, S., \& Mowlavi, N. 2004, A\&A, 418, 989

Pietrinferni, A., Cassisi, S., Salaris, M., \& Castelli, F. 2004, ApJ, 612, 168

Royer, F., Zorec, J., \& Gomez, A. E. 2007, A\&A, 463, 671

Schaerer, D., Charbonnel, C., Meynet, G., Maeder, A., \& Schaller, G. 1993, A\&\&AS, 102, 339

Schaller, G., Schaerer, D., Meynet, G., \& Maeder, A. 1992, A\&AS, 96, 269

Smith, N. \& Brooks, K.J. 2008, Handbook of Star Forming Regions, Vol. II, Bo Reipurth, ed, in press (arXiv:0809.5081)

Talon, S., Zahn, J.-P., Maeder, A., \& Meynet, G. 1997, A\&A, 322, 209

VandenBerg, D. A., Bergbusch, P. A., \& Dowler, P. D. 2006, ApJS, 162, 375

Ventura, P., Zeppieri, A., Mazzitelli, I., \& D'Antona, F. 1998, A\&A, 334, 953 
von Zeipel, H. 1924, MNRAS, 84, 665

Weidemann, V. 2000, A\&A A, 363, 647

Zahn, J.-P. 1992, A\&A, 265, 115

\section{Discussion}

K. Cover: Do you track the angular-momentum evolution of the star over its lifetime in your models? If so, how do you characterize the initial (or final) angular-momentum content of each model?

G. Meynet: Yes, the models self-consistently follow the evolution of the angular momentum in the interior. On the ZAMS, the star is supposed to have solid-body rotation, then under the action of convection, contraction/expansion, shear turbulence and meridional circulation, angular momentum evolves in each mass shell. Angular momentum may be lost at the surface as a result of stellar winds. It is to be noted that the efficiency of the angular-momentum transport depends on the angular velocity and the gradient of the angular velocity. Thus the rotation at a given time step is obtained by an iterative procedure. (This is what is meant by self-consistently above.)

C. Deliyannis: When you indicate that rotation increases the ages of massive stars by $25 \%$, do you include the effects of rotation on the convective core, and do you also include mixing (e.g., due to circulation) bringing in more hydrogen to the core?

G. Meynet: In the models I presented, the increase in the size of the convective core in the last part of the MS phase results from rotational mixing in the radiative zone. Rotational mixing provides fuel to the core by making $\mathrm{H}$ diffuse from the radiative envelope into the convective core. Rotational mixing also makes the He produced in the core diffuse into the radiative envelope, lowering its opacity.

S. VAUCLAIR: This is a comment about seismic constraints on core overshooting. With a graduate student we have studied in detail a solar-type star for which we have good seismic data, ( $\mu$ Arae), with the aim of constraining the size of the convective core, including overshooting. This is possible because the star is at the end of the main sequence, so that the core is He-rich, with a clear signature in the oscillation frequencies. We find that overshooting is very small or absent (less than $\alpha_{\mathrm{OS}}=0.02$; our paper is in preparation).

G. Meynet: This is a indeed a great result if it is possible from asteroseismology to make progress on the question of the size of the convective core (whose size may result from various processes, rotation and overshooting being two of them). In the transition mass range between stars with no convective core $\left(\mathrm{M}<1.1 \mathrm{M}_{\odot}\right)$ and stars with a welldeveloped convective core $\left(\mathrm{M}>1.3 \mathrm{M}_{\odot}\right)$ - these limits depend on metallicity - probably overshooting increases progressively, being nearly non-existent around a very small core and then reaching a moderate value above $\mathrm{M}>1.3 \mathrm{M}_{\odot}$. Therefore in the star you analyzed, if its mass is sufficiently small, I would not be surprised that very small overshooting is found.

A. DotTer: Why is the post-main sequence evolution of $\sim 10 \mathrm{M}_{\odot}$ solar-Z models (especially the extent of the blue loop) so strongly influenced by rotation?

G. Meynet: Rotation modifies the sizes of the cores (increasing them). The presence of blue loops and their extension depend sensitively on the size of the cores (the bigger they 
are, the shorter for instance are the blue loops; see the discussion in Maeder \& Meynet 2001, A\&A, 373, 555). Therefore the blue loop for a star of $9 \mathrm{M}_{\odot}$ at $Z=0.020$, computed with rotation is less extended than the blue loop for the same model without rotation. In the case of $12 \mathrm{M}_{\odot}$, the blue loop is even suppressed in the rotating models (see DHR in Meynet \& Maeder 2000, A\&A, 361, 101).

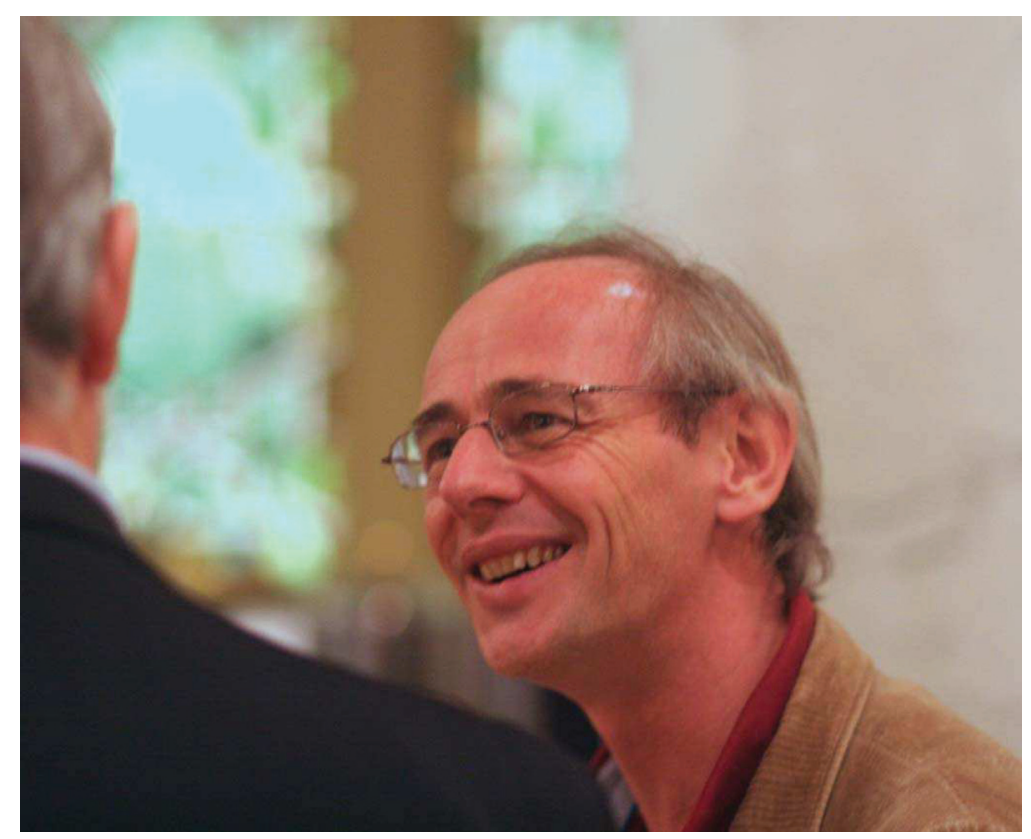

Georges Meynet 